Annuaire suisse de politique de développement

Partenariats public-privé et coopération internationale

\title{
Le partenariat public-privé dans le secteur de l'eau au Niger : autopsie d'une réforme
}

\section{Mahaman Tidjani Alou}

\section{(2) OpenEdition}

1 Journals

Édition électronique

URL : http://journals.openedition.org/aspd/360

DOI : 10.4000/aspd.360

ISSN : 1663-9669

Éditeur

Institut de hautes études internationales et du développement

\section{Édition imprimée}

Date de publication : 1 octobre 2005

Pagination : 161-177

ISSN : 1660-5934

\section{Référence électronique}

Mahaman Tidjani Alou, «Le partenariat public-privé dans le secteur de l'eau au Niger : autopsie d'une réforme », Annuaire suisse de politique de développement [En ligne], 24-2 | 2005, mis en ligne le 19 février 2010, consulté le 08 septembre 2020. URL : http://journals.openedition.org/aspd/360 ; DOI : https:// doi.org/10.4000/aspd.360 


\title{
Le partenariat public-privé dans le secteur de l'eau au Niger: autopsie d'une réforme
}

\author{
Mahaman Tidjani Alou*
}

es mutations intervenues dans le secteur de l'eau au cours de ces dernières années en République du Niger ${ }^{1}$ offrent un site particulièrement fécond pour l'étude du partenariat public-privé (PPP) tel qu'il se configure dans la plupart des pays d'Afrique de l'Ouest. Il faut reconnaître que dans le cas du Niger, ce partenariat s'inscrit dans le cadre des politiques de réformes économiques instaurées par les gouvernements qui se sont succédé au pouvoir depuis 1995. Celles-ci se sont traduites, entre autres, par l'enclenchement de la privatisation des entreprises publiques ${ }^{2}$. Mais ces réformes, qui intègrent aussi le secteur de l'eau, loin de se limiter aux seules entreprises publiques, s'élargissent également à l'hydraulique villageoise, qui fait face à de nouvelles dynamiques dans ses principes de gestion. Ces mutations, d'orientation résolument libérale, offrent un cadre propice pour comprendre le PPP tel qu'il prend forme dans le secteur de l'eau au Niger.

Bien entendu, seules les dimensions institutionnelles du problème seront envisagées $^{3}$. Ce qui exclut de fait les interrogations de type économique couramment utilisées et qui introduisent les questions liées aux effets économiques et financiers de la privatisation des entreprises publiques ${ }^{4}$. Notre orientation s'attache surtout à faire ressortir les transformations intervenues dans la gouvernance de l'eau au Niger, suite aux différentes réformes structurelles engagées au cours de cette dernière décennie $(1995-2005)^{5}$. Notre intérêt se focalisera plus particulièrement sur l'évolution du rôle de l'Etat en matière de gestion de l'eau, concomitamment à l'avènement de nouveaux acteurs non étatiques dans

* Politologue, enseignant chercheur à l'Université Abdou Moumouni, chercheur au Laboratoire d'études et de recherches sur les dynamiques sociales et le développement local (LASDEL).

1 Le Niger est un pays de l'Afrique sahélienne, couvrant une superficie de 1'267'000 km². Il est administrativement divisé en 8 régions, 36 départements et 265 communes. Sa population est estimée depuis le dernier recensement (2001) à plus 11 millions d'habitants. Il dispose d'un potentiel hydraulique considérable, comme l'attestent ses immenses ressources en eaux de surface et ses réserves en eaux souterraines. Voir Ministère de l'hydraulique et de l'environnement, Politiques et stratégies pour l'eau et l'assainissement. De l'eau pour un développement durable, Niamey, mai 2001.

2 S. Sidibé, La réforme du secteur parapublic au Niger, Niamey, Nouvelle Imprimerie du Niger, n.d.; Ministère des finances et du plan, Assemblée générale des entreprises publiques et parapubliques, rapport général, mars 1996.

3 C. Ménard, «Enjeux d'eau: la dimension institutionnelle», Revue Tiers Monde, $\mathrm{n}^{\circ}$ 166, avril-juin 2001, pp. 261-274 ; B. Freund, B. Lootvoet, «Où le partenariat public-privé devient l'instrument privilégié du développement économique et local. L'exemple de Durban, Afrique du Sud», Revue Tiers Monde, $\mathrm{n}^{\mathrm{o}} 181$, janvier-mars 2005.

4 Voir par exemple P. Plane, «La privatisation de l'électricité en Côte d'Ivoire : évaluation et interprétation des premiers résultats», Revue Tiers Monde, n 152, octobre-décembre 1997.

5 On peut considérer que c'est à partir de 1995 que le Niger renoue le dialogue avec le FMI, lors de la signature d'une lettre d'intention. 
l'arène hydraulique. Alors que pendant longtemps, l'Etat a joué un rôle prépondérant dans le secteur, aujourd'hui, il doit composer, à tous les niveaux de ses interventions, avec d'autres acteurs qui sont parties prenantes des nouvelles dynamiques de gouvernance dans le secteur de l'eau. Ainsi, nous nous attacherons à décrire le contenu de ce nouveau dispositif qui s'est mis en place et les nouvelles dynamiques institutionnelles qu'il induit autour de la ressource «eau», en traitant du nouveau positionnement de l'Etat dans un secteur où il était demeuré l'acteur unique jusqu'à la réforme. Nous nous intéresserons aussi à l'apparition de nouveaux acteurs non gouvernementaux (associations, entreprises) qui occupent désormais des espaces laissés vacants par l'Etat.

Ce travail nous situe au cœur de la problématique de l'Etat en Afrique. Mais l'angle qui sera privilégié concerne les politiques publiques ${ }^{6}$. Là encore, il ne s'agira pas de les évaluer et encore moins de mesurer leur performance. L'ambition ici sera d'identifier et de comprendre les acteurs que ces politiques engagent, les ressources qu'elles mobilisent, ainsi que les interactions et les nouveaux rapports qu'elles instituent dans le secteur de l'eau.

Envisagé dans cette approche, le PPP trouve un terrain d'expression intéressant, non pas à des échelles globales mais dans des secteurs d'activité bien identifiés de l'Etat, en l'occurrence le secteur de l'eau'. Dans cette perspective, notre recherche a porté aussi bien sur l'hydraulique urbaine que sur l'hydraulique villageoise, qui connaît également des transformations importantes.

Nous avons travaillé sur la documentation officielle disponible ${ }^{8}$ ainsi que sur divers articles de presse ${ }^{9}$ et sur des données recueillies au cours d'entretiens réalisés avec certains responsables du secteur eau au Niger ${ }^{10}$. Il va de soi que des enquêtes plus longues auraient permis d'apprécier davantage le fonctionnement en réel de ces institutions, de même que leur impact à partir d'une analyse plus fouillée des objectifs affichés par les politiques mises en œuvre.

Notre démarche s'articule autour de trois axes interdépendants: nous verrons d'abord, dans le cas qui nous concerne, que le PPP est l'expression d'une réforme dans le secteur de l'eau au Niger. Il convient dans ce sens de comprendre le contexte de cette réforme, ainsi que les raisons qui sont mises en avant pour la légitimer. Ensuite, il s'agira de décrire, dans leurs différents éléments constitutifs, les recompositions du paysage institutionnel né du PPP qui s'est mis en place dans le cadre de la nouvelle réforme. Enfin, on tentera de comprendre les implications de partenariat, telles qu'elles se donnent à voir dans le nouveau contexte.

B. Jobert, P. Muller, L'Etat en action. Politiques publiques et corporations, Paris, Presses Universitaires de France, 1987.

C. Ménard, op. cit.

Lois, ordonnances, décrets, décisions.

Nous avons consulté tous les articles et autres interviews parus dans la presse entre 1993 et 2001.

10 Nous tenons à remercier, pour avoir accepté de nous accorder des entretiens, Zibo Zakara, directeur du Programme sectoriel eau, Yvan Kédaj, assistant technique au Ministère chargé de l'hydraulique, André Patétat, directeur général de la Société d'Exploitation des Eaux du Niger (SEEN), et Atto Indatou, directeur d'exploitation de la Société de Patrimoine des Eaux du Niger (SPEN). Nous avons aussi consulté largement le site Internet de l'Autorité de régulation multisectorielle (ARM), <www.arm-niger.org>. 
La réforme du secteur de l'eau traduit d'abord la sanction d'une gestion publique défaillante et la définition d'un nouveau cadre d'intervention de l'Etat.

\section{L’Etat omniprésent}

Les modes de gestion de l'eau au Niger ont longtemps reposé sur la prévalence des services techniques de l'Etat. Ces derniers concevaient les politiques et les mettaient en œuvre, tant dans les zones urbaines que dans les zones rurales. Cette activité étatique s'est déployée selon des logiques purement techniciennes. Ainsi, pendant une longue période, tablant sur les besoins en eau supposés des populations, l'Etat a installé des infrastructures hydrauliques sur la base de ses politiques d'aménagement du territoire, sans toujours tenir compte de l'avis des populations vers lesquelles ces actions étaient orientées.

Dans les zones urbaines, c'est une entreprise publique qui s'est chargée d'assurer l'approvisionnement en eau potable des populations. Initialement accoudée à la compagnie d'électricité (la Société Anonyme pour la Fourniture d'Electricité [SAFELEC] puis la Société Nigérienne d'Electricité [NIGELEC]), la gestion de l'hydraulique urbaine s'autonomise à partir de 1987 avec la création de la Société Nationale des Eaux $(\mathrm{SNE})^{11}$. Il s'agissait alors de donner plus de visibilité à la gestion d'un secteur qui évoluait sans aucune autonomie. D'ailleurs, l'Etat lui-même avait totalement délaissé le secteur à la discrétion de la compagnie d'électricité. Dans ces conditions, il ne pouvait guère espérer un quelconque appui financier pour développer le secteur dans un contexte de développement urbain galopant. Ainsi, la création de la SNE, sur crédit de la Banque mondiale, se présentait dans son contexte comme une réponse au besoin d'investissement de ce secteur stratégique qui s'avérait de plus en plus incapable de prendre en charge l'approvisionnement en eau des populations urbaines.

En zone rurale, l'approvisionnement en eau potable des populations revenait à l'OFEDES ${ }^{12}$, une autre entreprise publique, créée par l'Etat. Cette entreprise avait une compétence exclusive qui se déclinait en plusieurs dimensions:

11 La SNE a été créée en 1987 par l'Ordonnance n 87-31 du 24 septembre 1987, portant création de la Société Nationale des Eaux (SNE). Son siège social est à Niamey. Voir Ministère de l'hydraulique et de l'environnement, Programme hydraulique Suisse Niger, Le rôle de l'Etat dans la gestion des ressources naturelles. Recueil des textes législatifs et réglementaires, Niamey, 1999. La SNE dispose de représentations dans tout le pays. Elle est une propriété exclusive de l'Etat du Niger. Elle comptait au moment de sa privatisation 540 employés, 51 centres, 8 stations de pompage, 3 stations de traitement des eaux, 85 réservoirs et 124 forages en service. Son réseau s'étalait sur $1600 \mathrm{~km}$. Elle disposait aussi de 1400 bornes fontaines et de 2 usines d'eau. Voir Le Républicain, 14/12/2000, p. 8.

12 L'OFEDES est un établissement à caractère industriel et commercial, créé en 1963 en vue de la réalisation des forages et des puits. Voir Alternative Magazine, $\mathrm{n}^{\circ}$ 00, 24/04/1996; voir aussi l'Ordonnance n 74 -32 du 15 novembre 1974 portant création d'un établissement public chargé de l'exploitation des eaux du sous sol. Cette ordonnance modifie la Loi 61-31 du 7 mai 1963 portant création d'un établissement public chargé de l'exploitation des eaux de sous-sol dans la République du Niger, modifiée elle-même par la Loi 73-14 du 5 avril 1974. 
- assurer le fonçage des puits en investissement humain ${ }^{13}$ ou en régie ${ }^{14}$, ainsi que l'exécution des forages en zone urbaine et pastorale;

ـ assurer l'exploitation, c'est-à-dire l'entretien et le fonctionnement de puits et forages situés en zone rurale et pastorale ainsi que de leur superstructure ;

- effectuer la réalisation des réseaux de distribution d'eau potable dans les centres secondaires.

Sur cette base ${ }^{15}$, l'OFEDES contrôlait la production et la distribution de l'eau en zone rurale, ainsi que l'entretien des infrastructures hydrauliques qu'elle installait $^{16}$. Là encore, c'est une dynamique étatique qui a prévalu. La diffusion de ces infrastructures devait permettre d'assurer à terme l'approvisionnement en eau des populations rurales nigériennes.

Ainsi, tout au long des années 1960 et 1970, l'Etat jouait un rôle central dans le secteur hydraulique. D'ailleurs, nul ne lui contestait ce rôle qu'il s'était attribué, qui correspondait aux perceptions du moment en matière d'action publique: l'Etat voulait tout faire. Et l'idée que l'Etat puisse être un acteur central du développement économique et social était largement partagée. Ce que nous observons là dans le secteur hydraulique s'étendait à bien des secteurs qui devenaient de fait des champs d'intervention naturels de l'Etat. Mais cette prévalence de l'Etat dans le secteur hydraulique montra vite ses limites.

\section{Schéma 1: Le secteur de l'eau avant le PPP}

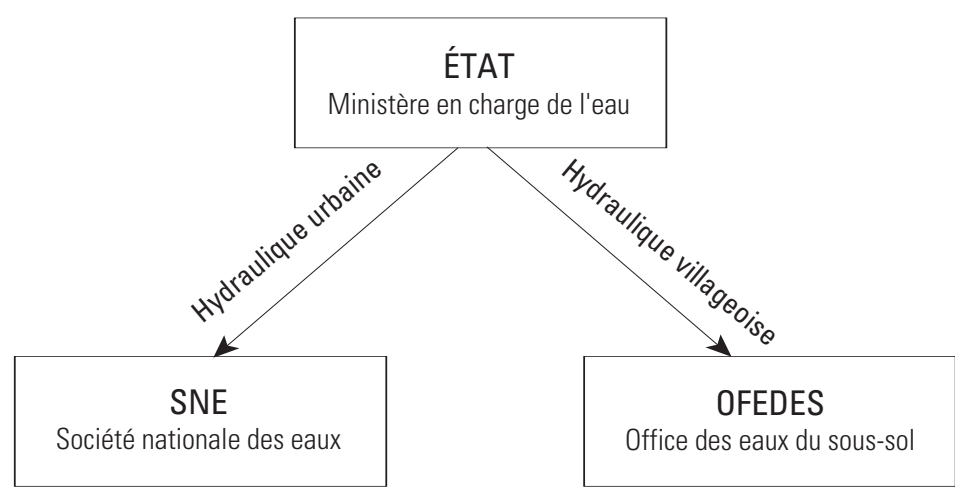

13 L'investissement humain fait référence à la participation des populations dans la réalisation des ouvrages hydrauliques.

14 Ici, la régie fait référence à la capacité de l'OFEDES de contracter directement en faisant des recettes au titre des travaux qu'elle réalise.

15 Ordonnance $\mathrm{n}^{\circ}$ 74-32 du 15 novembre 1974, op. cit.

16 Voir à ce sujet la Loi 94-21 du 6 septembre 1994 portant création d'un établissement public à caractère industriel et commercial dénommé Office des eaux du sous-sol (OFEDES). Cette loi confirme la position prépondérante de l'OFEDES dans le secteur de l'hydraulique rurale. 


\section{L’Etat délégitimé}

L'omniprésence de l'Etat, en dépit des investissements importants qu'elle a générés, s'est avérée peu porteuse. Les politiques qui avaient été mises en place n'ont pas eu les effets escomptés sur le développement du secteur de l'eau. La mauvaise gestion s'est propagée et s'est partout érigée en règle. Mais tant que l'Etat et la communauté internationale pouvaient prendre en charge les énormes investissements que nécessitait le secteur hydraulique, ces dysfonctionnements n'étaient pas visibles. L'effritement du modèle intervient avec la crise des finances publiques, amplifiée par l'amenuisement du financement international, consécutive au refus par le Niger de signer son deuxième programme d'ajustement structurel, à la veille des soubresauts qui ont précédé la Conférence nationale de 1991.

Cette crise fait apparaître sous un nouveau jour les modes de gestion publique de l'eau tant dans les villes que dans les campagnes. Elle révèle les limites du modèle qui avait prévalu depuis l'indépendance. En effet, comme nous l'avons souligné plus haut, l'Etat, à travers ses structures, s'était montré incapable d'assurer la bonne gestion du secteur en charge de l'eau. La SNE s'est vite révélée dépendante des perfusions financières d'un Etat incapable d'honorer les factures d'eau de ses administrations. En ce qui concerne l'hydraulique rurale, c'est surtout l'inopérationnalité du système de maintenance mis en place qui posait problème (gestion communautaire, participation des collectivités territoriales), dans un contexte marqué par le retrait de l'Etat en raison de l'amenuisement de la rente de l'uranium et le tarissement du financement international tel qu'il poignait à la fin des années 1980. Il est vite apparu que les nombreux ouvrages hydrauliques construits par l'Etat au cours des trois décennies qui ont suivi l'indépendance n'étaient guère durables. Les problèmes de leur gestion, avec leurs répercussions sur la maintenance des ouvrages hydrauliques, n'étaient pas étrangers à la situation.

De fait, on peut affirmer que c'est la crise de l'Etat nigérien à partir de la fin des années 1980 qui fut à la base des transformations qui se sont opérées dans les modes de gestion de l'hydraulique. Participant de la mouvance générale des politiques d'ajustement structurel, ces transformations ont provoqué le transfert d'une bonne partie des prérogatives naguère relevant de l'Etat vers le secteur privé et les communautés villageoises. Ce transfert de compétence de gestion, né de la crise de l'Etat, a aussi été favorisé par les bailleurs de fonds du Niger, qui ont saisi cette situation de mollesse structurelle de l'Etat pour le contourner, en promouvant, pour accompagner leur financement, de nouvelles règles de gestion des ressources hydrauliques. Des textes législatifs et réglementaires sur le régime de l'eau ${ }^{17}$, adoptés au cours des années 1990, vont fournir la base juridique nécessaire à l'émergence d'un nouveau dispositif institutionnel.

17 Une ordonnance sur le régime de l'eau (Ordonnance $n^{\circ}$ 93-014) a été adoptée en 1993 et modifiée en 1998. Elle se veut un cadre juridique général qui organise le secteur de l'eau au Niger. 
Vers de nouvelles politiques de l'eau:

l'émergence d'un partenariat public-privé

Les nouvelles politiques de l'eau, qui se sont traduites par la mise en place d'un PPP, trouvent leur fondement dans une crise profonde de gestion, tant au niveau de l'hydraulique urbaine qu'au niveau de l'hydraulique rurale. Elles interviennent dans un contexte de déficit d'investissement public dans le secteur de l'eau et se veulent une réponse au constat d'échec des politiques jusque-là mises en œuvre. A cet égard, il convient de relever que les bailleurs de fonds ont conditionné de plus en plus leurs interventions dans le secteur par des exigences de réforme. Dans le domaine de l'hydraulique urbaine, ces exigences sont allées d'abord dans le sens d'une restructuration de la SNE, puis ont évolué vers sa privatisation. En effet, c'est en octobre 1996 que le gouvernement décida d'inscrire cette entreprise publique sur la liste des entreprises à privatiser ${ }^{18}$, suite à la mission effectuée par un consultant de la Banque mondiale. Au niveau de l'hydraulique villageoise, c'est la gestion participative qui a fait son apparition dans les arènes locales, encouragée par le changement d'orientation des bailleurs de fonds du secteur ${ }^{19}$. Partout, l'installation des infrastructures hydrauliques fut conditionnée par la participation des populations à la construction et à la gestion des ouvrages ainsi que par l'adoption de modes de gestion plus ouverts (gestion communautaire, gestion déléguée), où l'Etat ne joue plus le rôle central qui lui était naguère reconnu.

Deux lettres de politique sectorielle furent adressées par le gouvernement nigérien à la Banque mondiale pour donner un cachet officiel aux nouvelles orientations. C'est ainsi qu'au niveau de l'hydraulique, le gouvernement s'est engagé à promouvoir des réformes qui réajusteraient la place de l'Etat dans la gestion du secteur. Parmi les axes de la nouvelle politique de l'eau, en son volet urbain, on retiendra plusieurs points qui attestent du transfert des compétences de l'Etat vers d'autres acteurs:

$\checkmark$ «l'implication et la responsabilisation de collectivités locales dans la gestion du secteur, en faisant procéder au transfert de certains des domaines de compétence de l'Etat aux collectivités locales décentralisées;

$\checkmark$ la promotion du secteur privé et de la société civile pour la gestion du secteur par le renforcement de leurs capacités dans la prise en charge de la maîtrise de l'œuvre des ouvrages et infrastructures hydrauliques;

- la pleine participation des populations à la conception et à la réalisation des travaux, l'amélioration de la prise en charge des infrastructures, la clarification et le respect des rôles des différents partenaires (Etat, collectivités, secteur privé, populations bénéficiaires) et la sécurisation des droits d'exploitation $»^{20}$.

18 Ordonnance ${ }^{\circ}$ 96-062 du 22 octobre 1996.

19 Voir leur liste dans A. Hassane, A. Karadji, F. Balmer (dir.), Bien gérer l'eau du Sahel. Regards sur vingt ans de coopération entre la Suisse et le Niger dans le secteur de l'hydraulique, Berne, Direction du développement et de la coopération (DDC); Niamey, Ministère de l'hydraulique et de l'environnement; Genève, IUED, 1996.

20 Voir Ministère du plan, Ministère des ressources en eau, Lettre de politique sectorielle de l'hydraulique urbaine, Niamey, 08/03/2001, p. 2. 
Cette tendance de l'Etat à s'ouvrir vers d'autres acteurs est également visible dans le secteur de l'hydraulique rural. En effet, on trouve parmi les objectifs affichés dans ce secteur des orientations relatives à «la promotion du secteur privé et la pleine participation de la société civile (associations d'usagers) dans la gestion et la prise en charge de la maîtrise d'œuvre déléguée des ouvrages et infrastructures hydrauliques $»^{21}$.

Ainsi, à partir de 2001, c'est toute la politique de l'eau conduite par l'Etat nigérien qui s'articule sur le PPP. Cette politique s'inscrit dans un processus plus global de réformes et vise de manière plus spécifique les objectifs suivants:

$\square$ améliorer les performances techniques et financières du sous-secteur;

口 assurer une gestion commerciale efficiente ;

$\checkmark$ accroître le taux de desserte en eau potable orienté en particulier vers les populations à faible revenu ;

ـ assurer à moyen terme l'équilibre financier du sous-secteur ${ }^{22}$.

Cette politique se présente comme une réponse aux nombreux problèmes auxquels l'Etat fait face dans le secteur hydraulique. Et pour donner une forme concrète à cette politique, une nouvelle architecture institutionnelle traduit désormais le nouveau partenariat.

\section{Le partenariat public-privé} comme expression d'un nouveau cadre institutionnel

La réforme du secteur de l'eau au Niger s'inscrit dans le cadre du programme d'ajustement structurel. Elle induit une définition et une articulation nouvelles des rôles des acteurs impliqués dans la gestion de l'eau. On assiste ainsi à une recomposition de l'arène hydraulique dans son ensemble, mettant à jour une nouvelle configuration du secteur.

\section{Le nouveau schéma de la gouvernance de l'eau en zone urbaine}

La nouvelle gouvernance de l'eau, issue de la réforme du secteur, s'est traduite par la mise en place de nouvelles structures. D'abord, en zone urbaine, l'arène hydraulique dans laquelle l'Etat occupait une place centrale s'est élargie à de nouveaux opérateurs, qui ont donné ses contours institutionnels actuels à la politique de privatisation dans le secteur de l'eau. En outre, une structure d'accompagnement a été mise en place pour appuyer la réforme.

\section{Les nouveaux opérateurs: un dispositif quadripolaire}

La nouvelle configuration de l'hydraulique urbaine se structure autour de quatre pôles: l'Etat, la Société de Patrimoine des Eaux du Niger (SPEN), la Société d'Exploitation des Eaux du Niger (SEEN) et l'Autorité de régulation multisectorielle (ARM).

21 Ibid., p. 3.

22 Voir à ce sujet l'étude, commandée par la SEEN, Assistance du maître d'ouvrage «SPEN》 à la réalisation des bornes fontaines, rapport final, septembre 2004, p. 2. 


\section{L'Etat}

Malgré sa présence incontestable dans le secteur, les compétences de l'Etat se restreignent à des fonctions régaliennes bien identifiées. A cet égard, un accord règle les rapports entre l'Etat et ses nouveaux partenaires dans la gestion publique de l'eau au Niger ${ }^{23}$. L'Etat «définit la politique sectorielle, la gestion des ressources en eau et en élabore le cadre législatif et réglementaire. Il établit la politique tarifaire ${ }^{24}$. Par ailleurs, l'Etat crée une nouvelle société à laquelle il concède ses anciennes attributions. En outre, en sa qualité de plus grand consommateur d'eau et de gros débiteur de l'ancienne SNE, il devra désormais payer ses factures à bonne date et rationaliser davantage la consommation d'eau de ses administrations.

\section{La Société de Patrimoine des Eaux du Niger (SPEN)}

La SPEN est une société d'Etat créée le 14 août 2000 dans le cadre de la réforme et à laquelle l'Etat transfère, par contrat de concession, «la gestion physique, comptable et financière des biens et des droits immobiliers du domaine public naturel et artificiel du sous-secteur de l'hydraulique urbaine et semiurbaine. Le domaine public naturel concerne les cours d'eau, lacs, étangs et sources, les nappes souterraines, les sources thermales et minérales. Quant au domaine public artificiel, il vise les ouvrages hydrauliques réalisés pour compte et usage des collectivités publiques et les points aménagés à l'usage du public $\gg^{25}$. C'est donc un vaste domaine qui est concédé à la SPEN, chargée d'assurer désormais la gestion du patrimoine de l'Etat dans le secteur de l'hydraulique urbaine et semi-urbaine. «A ce titre, la SPEN, à travers le contrat de concession, est responsable de la réalisation de tous les investissements, de la recherche de fonds pour financer ces investissements. Elle est maître d'œuvre et maître d'ouvrage des travaux de réhabilitation des infrastructures hydrauliques; elle est en outre chargée de la sensibilisation du public sur l'économie et sur l'hygiène de l'eau. ${ }^{26}$ Les rapports entre l'Etat et la SPEN sont réglés par le contrat-plan annexé à l'accord qui régit les parties.

\section{La Société d'Exploitation des Eaux du Niger (SEEN)}

La SEEN est une société de droit privé nigérien mise en place le $1^{\text {er }}$ juin 2001 dans le cadre de la politique de privatisation de l'Etat. Elle est chargée, par un contrat d'affermage, de la production, du transport et de la distribution de l'eau dans les centres urbains et semi-urbains. Au total, ce sont les 51 centres qu'exploitait la SNE qui passent sous son contrôle ${ }^{27}$. Il convient d'ajouter que la SEEN assure aussi l'exécution de certains travaux en vue de l'amélioration de la qualité du service affermé de la distribution de l'eau ${ }^{28}$. C'est autour de la SEEN

23 Voir le contrat de concession entre la République du Niger et la Société de Patrimoine des eaux du Niger (SPEN), signé le 20 mars 2001.

24 Loi 2000-12 du 14 août 2000, portant réorganisation de l'activité de production, transport et distribution de l'eau dans le sous-secteur de l'hydraulique urbaine et créant la Société de Patrimoine des Eaux du Niger (SPEN), article 2.

25 Ibid., article 10.

26 Seyni Salou, directeur général de la SPEN, interview accordée au Sahel Dimanche, 07/12/2001, p. 4.

27 Depuis lors, un nouveau centre s'est ajouté aux 51 centres initiaux. Il faut penser ici la mise en affermage comme un processus dynamique, étant donné la possibilité qui est ouverte d'élargir le périmètre affermé.

28 ARM, <www.arm-niger.org > >Eau > Le secteur de l'eau au Niger. 
que s'articule le PPP tel qu'il prend forme au Niger, étant donné la nature semipublique des autres acteurs de l'arène hydraulique. La création de la SEEN marque l'entrée des privés dans la gestion de l'eau au Niger. Son actionnaire principal est la société Véolia, l'ancien Vivendi Water. Elle détient $51 \%$ du capital de la SEEN. 34\% des actions ont été vendues à des privés nigérien et $10 \%$ au personnel de l'ancienne SNE. L'Etat, pour sa part, ne détient que 5\% du capital de la nouvelle société conduite par un opérateur de renommée mondiale $^{29}$. Ainsi, le partenariat mis sur pied n'est pas classique, puisqu'il s'agit d'un privé international, professionnel avéré de l'hydraulique, paré d'une solide réputation dans le monde, et donnant par conséquent à la nouvelle configuration une tout autre épaisseur. La SEEN exerce ses activités sous le contrôle de la SPEN, qui assure le suivi de la réforme, et d'une autorité de régulation, selon des règles préétablies dans le contrat de performance annexé au contrat de concession.

\section{L'Autorité de régulation multisectorielle (ARM)}

L'ARM constitue le dernier élément du dispositif. Bien qu'elle ait été créée depuis $1999^{30}$, elle n'a été installée de façon effective qu'en $2003^{31}$, bien après la mise en place de la SPEN et de la SEEN. L'ARM est «une personne morale de droit public, indépendante, dotée de l'autonomie financière et de gestion. Ses décisions ont le caractère d'actes administratifs, elles sont susceptibles de recours juridictionnel $»^{32}$. Elle «est chargée de la régulation des activités exercées sur le territoire du Niger dans les secteurs de l'eau, de l'énergie, des télécommunications et du transport ${ }^{33}$. Ses missions de régulation couvrent plusieurs domaines:

ऽ «veiller à l'application des textes législatifs et réglementaires régissant les secteurs dans des conditions objectives, transparentes et non discriminatoires;

- protéger les intérêts des utilisateurs et des opérateurs, en prenant toute mesure propre à garantir l'exercice d'une concurrence saine et loyale dans le secteur, dans le cadre des dispositions légales et réglementaires en vigueur;

๖ promouvoir le développement du secteur en veillant notamment à l'équilibre économique et financier et à la préservation des conditions économiques nécessaires à sa viabilité;

$\checkmark$ mettre en œuvre les mécanismes de consultation des utilisateurs et des opérateurs prévus par les lois et règlements $»^{34}$.

L'ARM dispose de pouvoirs de sanction qui s'exercent «soit d'office, soit à la demande d'une organisation professionnelle, d'une association d'utilisateurs ou

29 M. Laimé, «Main basse sur l'eau des villes», Le Monde diplomatique, mars 2005, pp. 16-17. Voir aussi «Saga Vivendi Universal: un rêve en échec», Le Monde: Dossiers et Documents, n 320, mai 2003.

30 Ordonnance $\mathrm{n}^{\circ}$ 99-044 du 26 octobre 1999 portant création, organisation et fonctionnement d'une autorité de régulation multisectorielle.

31 Selon les propos tenus par Seyni Salou, directeur général de la SPEN, dans l'interview déjà citée, elle est opérationnelle depuis 2001, mais on lit dans Le Régulateur ( $\mathrm{n}^{\circ} 00$, décembre 2004, p. 5) que les membres du Conseil national de régulation n'ont prêté serment qu'en décembre 2003.

32 Ordonnance ${ }^{\circ}$ 99-044, article 1, paragraphe 2.

33 Ibid., article 2, paragraphe 1.

34 Ibid., article 2, paragraphe 3. 
d'une personne physique ou morale ayant intérêt à agir ${ }^{35}$. Elle détient aussi des fonctions consultatives vis-à-vis des autorités et a un devoir d'information vis-àvis du public, notamment à travers son bulletin officiel, Le Régulateur, et son site Internet.

Elle est dirigée par le Conseil national de régulation (CNR), qui comprend cinq membres: un président et quatre directeurs sectoriels. Dans le secteur de l'eau, elle agit à travers le directeur sectoriel eau, qui, comme ses pairs, est recruté par appel à candidatures. C'est une innovation qui mérite d'être soulignée et qui est de nature à créer les conditions de l'indépendance de la nouvelle autorité.

Selon la présidente du Conseil national de régulation, l'ARM a été créée «pour émettre un signal fort de transparence dans la gestion postprivatisation à l'endroit des différents acteurs impliqués dans le processus de privatisation ${ }^{36}$. Ce point de vue rejoint celui déjà exprimé par le directeur général de la SPEN, qui voit dans l'ARM «un arbitre entre les acteurs du secteur de l'eau [...], qui joue un rôle de stabilisation, notamment entre l'Etat et la SPEN, entre la SPEN et la SEEN, entre la SEEN et les consommateurs, avec toutes les règles de jeu: concurrence loyale, transparence, respect des contrats signés ${ }^{37}$.

Ce nouveau dispositif est appuyé dans sa mise en œuvre par le Projet sectoriel eau.

\section{-Une structure d'accompagnement: le Projet sectoriel eau (PSE)}

Le PSE répond au besoin d'investissement exprimé dans le secteur eau. Les politiques de réforme engagées étaient une conditionnalité d'accès aux crédits obtenus dans le cadre de la mise en œuvre du projet. Il est financé par quatre bailleurs de fonds, à savoir la Banque mondiale (BM), l'Agence française de développement (AFD), la Banque ouest africaine de développement (BOAD) et le gouvernement chinois. Son montant est estimé à environ 80 millions d'euros. Ce projet, qui couvre la période 2002-2006, a entre autres objectifs d'appuyer le processus de réforme engagé dans le secteur de l'hydraulique au Niger, en finançant les investissements en vue d'accroître la desserte en eaux dans les 51 centres gérés par la SEEN. Il vise aussi à élaborer et à clarifier les textes législatifs et réglementaires portant sur le régime de l'eau ${ }^{38}$.

Plusieurs activités sont programmées dans sa composante hydraulique urbaine:

$\checkmark$ réhabilitation, extension des installations de production, transport et distribution dont l'exécution est assurée par la SEEN;

$\checkmark$ programmation des petites extensions et branchements sociaux par la SEEN dans le cadre du marché de travaux associé au contrat d'affermage;

$\square$ construction de bornes fontaines confiée également à la SEEN ${ }^{39}$.

35 Ibid., article 4, paragraphe 4.1.

36 Interview de Boubacar Sory Zalika, Le Régulateur, nº 00, décembre 2004, p. 4.

7 Interview déjà citée de Seyni Salou, directeur général de la SPEN.

8 Interview de Zibo Zakara, directeur du PSE, Sahel Dimanche, 06/08/2004.

9 Voir à ce sujet l'étude déjà citée de la SEEN, p. 2. 
Schéma 2: Le PPP dans le secteur de l'eau au Niger - Hydraulique urbaine

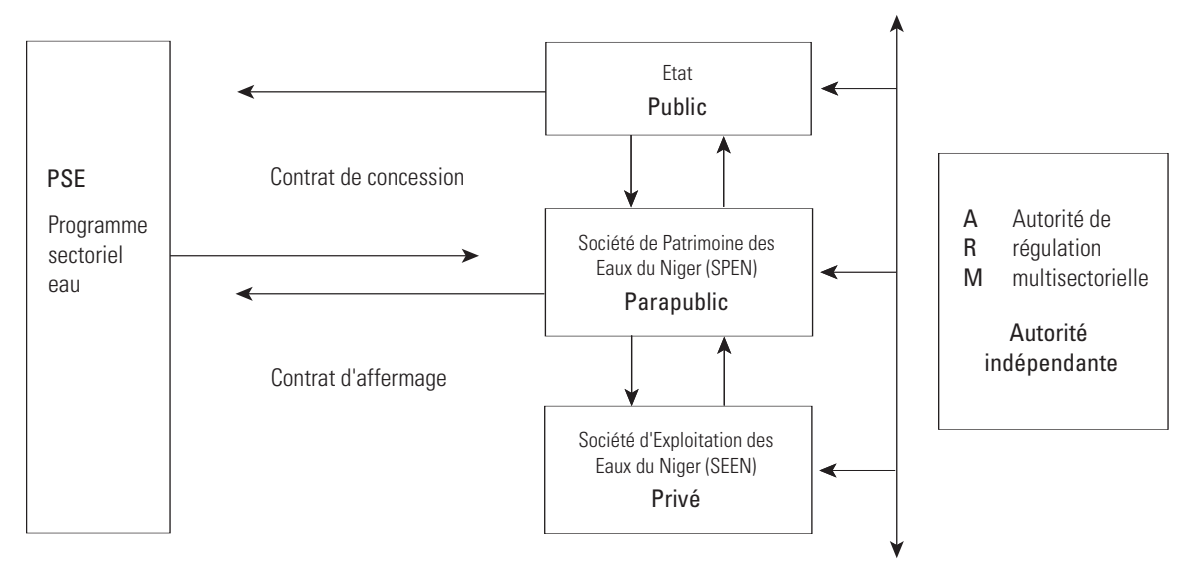

\section{Emergence d'acteurs privés dans la gestion de l'hydraulique villageoise}

L'hydraulique villageoise est également concernée par les mutations qui affectent la gestion publique de l'eau. Qu'elle ait été assurée par l'Etat ou par les communautés villageoises, cette gestion s'est avérée le plus souvent défaillante. Elle n'a guère réussi à créer les conditions d'une gestion durable des ouvrages hydrauliques ${ }^{40}$, d'où l'intervention croissante sur ce terrain de nouveaux acteurs privés par le biais de la gestion déléguée. Celle-ci avait déjà été prévue par les textes en vigueur ${ }^{41}$. En l'occurrence, il s'agit de concéder la gestion des ouvrages hydrauliques à des acteurs privés, moyennant redevances. Ce mode de gestion a tendance aujourd'hui à se diffuser, notamment en ce qui concerne les mini-adductions d'eau potable (mini-AEP).

Deux types d'opérateurs interviennent dans le secteur:

- les opérateurs individuels, qui sont soit des commerçants, soit des techniciens de l'eau reconvertis dans la gestion des mini-AEP;

- les opérateurs qui s'organisent en société pour assurer la gestion de l'eau dans plusieurs villages à la fois.

En fait, c'est un secteur marchand plus développé et plus structuré qui émerge dans les villages. Alors qu'il n'y a pas si longtemps la vente de l'eau en zone rurale relevait presque du tabou, elle paraît aujourd'hui totalement admise ${ }^{42}$. Les opérateurs qui s'y adonnent sont plus en plus nombreux, tant le commerce de l'eau au village se présente comme une activité fortement lucrative.

40 A. Elhadj Dagobi, J.-P. Olivier de Sardan, «La gestion communautaire sert-elle l'intérêt public? Le cas de l'hydraulique villageoise au Niger», Politique africaine, $\mathrm{n}^{\circ} 80,2000$, pp. 153-168.

41 Ordonnance $n^{\circ}$ 93-014 du 2 mars 1993 portant régime de l'eau, modifiée par la Loi no 98-041 du 7 décembre 1998.

42 Voir l'interview de Zibo Zakara, à l'époque directeur des infrastructures hydrauliques au Ministère de l'hydraulique et de l'environnement, Sahel Dimanche, 31/12/1998. 
Ici, le PPP prend une forme particulière. Il passe par les communes. On n'a pas affaire, comme en zone urbaine et semi-urbaine, à un partenaire de grande taille, à l'image de la SEEN, mais à de nombreuses associations d'usagers de l'eau qui, à leur tour, délèguent la gestion de l'ouvrage hydraulique en question à un opérateur privé dans des conditions particulières prévues par la loi. En fait, pour la gestion des mini-adductions d'eau potable, il est prévu un contrat type entre la commune, l'association des usagers de l'adduction d'eau et la société délégataire, qui peut être un commerçant, un groupement d'intérêt économique (GIE), une société unipersonnelle, une société à responsabilité limitée (S. à r.l.) ou une société anonyme (SA). On voit bien que la palette des acteurs concernés est assez ouverte. Bien entendu, ce cadre contractuel part du principe qu'il existe une convention entre la collectivité territoriale et l'association des usagers de l'eau. Il précise les conditions de transfert de certaines compétences spécifiques en matière de gestion du service de l'eau. La délégation est concédée par arrêté du ministre chargé de l'hydraulique pour une durée de trois ans. Le contrat précise les obligations de chacun des partenaires impliqués dans la gestion de l'ouvrage hydraulique.

Dans ce cas de figure, le délégataire est le personnage central. C'est lui qui symbolise le privé. Il a pour rôle d'assurer toutes les fonctions de l'exploitation de la mini-adduction d'eau potable à la satisfaction des consommateurs, par la gestion des installations qui lui sont confiées. A ce titre, il assure l'entretien du forage, la maintenance des installations autres que le forage, la distribution de l'eau potable aux usagers, la promotion de bonnes relations avec les consommateurs. Par ailleurs, il prend en charge les dépenses d'exploitation et procède au paiement régulier d'une redevance à l'association des usagers. Le délégataire est en outre assujetti à de nombreuses autres obligations énoncées dans le contrat.

L'association, pour sa part, a pour mission de garantir le bon fonctionnement des installations qu'elle a reçues en charge de la part de la collectivité territoriale, en fournissant au délégataire les appuis nécessaires à l'accomplissement de ses tâches dans les meilleures conditions possible. A ce titre, elle assure entre autres des fonctions de représentation, de protection des sites, etc.

La commune, qui a reçu de l'Etat la propriété des infrastructures, a pour mission de satisfaire les besoins de la population en collaborant avec les différents acteurs impliqués et en assurant des fonctions de contrôle quant à la qualité du service.

L'Etat, à travers les services déconcentrés du ministère en charge de l'hydraulique, est désormais relégué à des fonctions d'assistance diverses liées à l'archivage et à l'examen des états de gestion de l'exploitation ainsi qu'aux informations techniques concernant la ressource en eau. Il assure aussi des fonctions d'appui-conseil et participe au besoin au règlement des conflits liés à l'exploitation qui peuvent subvenir au cours du mandat du délégataire. 
Schéma 3: Le PPP dans le secteur de l'eau au Niger - Hydraulique villageoise

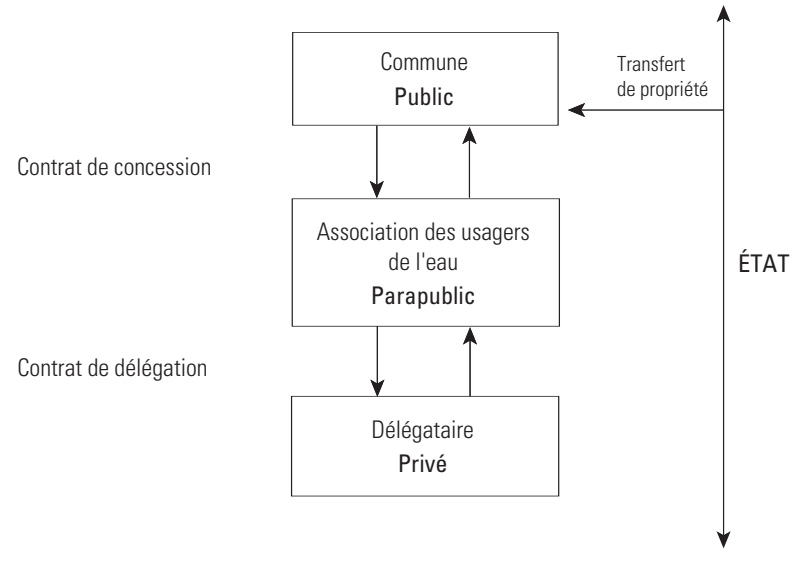

\section{Le partenariat public-privé dans le secteur de l'eau au Niger: les hypothèques}

En fin de compte, le PPP, tel qu'il s'institutionnalise au Niger, se développe selon une logique multiforme, mettant au jour des stratégies variées et montrant les modalités de transformation du rôle de l'Etat, qui fait face désormais à de nouveaux acteurs, eux-mêmes engagés dans des processus d'apprentissage où l'enjeu pour eux est avant tout de s'affirmer dans un nouveau contexte institutionnel.

Dans le secteur de l'hydraulique urbaine et semi-urbaine, le PPP s'inscrit dans la dynamique de la privatisation des entreprises publiques telle qu'elle est prônée par la Banque mondiale, leader des bailleurs de fonds en la matière. C'est un formatage classique, qui met en relation de complémentarité des acteurs étatiques et des acteurs privés, sous l'arbitrage d'une autorité de régulation indépendante. Ici, il ne s'est pas agi de vendre une société d'Etat, comme l'avaient suggéré les débats au moment du lancement de la réforme. Cette perception était identifiable notamment dans le discours syndical ${ }^{43}$, où la privatisation a été systématiquement assimilée à la vente du patrimoine public. L'option retenue a privilégié l'affermage de l'exploitation de l'eau par une société privée, créée pour la circonstance. Ainsi, la nouvelle société contrôlée par Véolia reprend toutes les activités liées à la production et à la vente de l'eau dans les zones urbaine et semi-urbaine. C'est donc un commerce fort rentable que l'Etat rétrocède au profit d'un opérateur qui jouit d'une incontestable réputation à l'échelle internationale.

Mais l'Etat maintient la propriété de son patrimoine, qu'il concède à une société publique (SPEN) créée dans la foulée de la réforme. Celle-ci reprend la gestion du patrimoine de l'Etat et devient du coup le principal interlocuteur de

43 Voir l'interview du secrétaire général de l'Union des syndicats des travailleurs du Niger (USTN), Le Démocrate, 24/02/1997, p. 3. 
la nouvelle société (SEEN). La SPEN est aussi responsable de l'investissement public en la matière et se charge en conséquence de la gestion des redevances perçues auprès de la SEEN et des relations avec les bailleurs de fonds qui ont conditionné l'accès à leurs crédits par la mise en œuvre de la réforme. C'est à ce titre que la Société de Patrimoine des Eaux du Niger est devenue le principal interlocuteur du PSE.

En fait, le schéma est assez complexe puisqu'il associe l'Etat (public), une société publique (parapublic), une société privée (privé) et une autorité indépendante (parapublic) chargée de garantir le respect des nouvelles dispositions juridiques.

En matière d'hydraulique villageoise, le schéma adopté ne couvre pas l'intégralité des ouvrages hydrauliques. Il concerne surtout la gestion des mini-AEP, qui fonctionnaient pour la plupart selon une logique de gestion communautaire. Dans ce cas de figure, l'Etat maintient des fonctions de conservation de documents et d'appui-conseil sur divers volets. Il fournit parfois des prestations, mais il est quasiment absent de l'arène locale. Le nouvel acteur qui émerge est la commune. Elle est issue du processus de décentralisation au Niger, qui s'est traduit par l'installation de 265 communes $^{44}$. On peut considérer que la commune est bien un acteur public, tout comme l'Etat l'est au niveau national. C'est elle qui détient la propriété des ouvrages hydrauliques qu'elle concède à l'association d'usagers de la mini-AEP. Jusqu'à une date récente, c'est un comité de gestion qui jouait ce rôle. Le choix de créer des associations d'usagers permet aux communautés locales d'agir désormais dans un cadre formel. L'association d'usagers entre à son tour en partenariat avec le privé à travers un contrat de délégation de gestion qui prend la forme d'un «affermage à périmètre réduit» puisqu'il s'exerce à l'échelle de la mini-AEP. Enfin, le dispositif ne prévoit pas d'autorité de régulation. Ce sont les services techniques déconcentrés de l'Etat qui jouent en quelque sorte ce rôle.

Dans un cas comme dans l'autre, l'Etat est présent mais il exerce des fonctions différentes. De même, la forme du privé change selon qu'on est en zone urbaine ou en zone rurale. Mais dans les deux cas, l'Etat est dépossédé de larges pans des compétences qu'il exerçait naguère au profit d'acteurs nouveaux, tant publics que privés.

En dépit de la pluralité des acteurs qu'elle associe, cette nouvelle configuration reste fonctionnelle. Sa dynamique actuelle doit beaucoup au fait qu'elle conditionne l'accès aux ressources financières importantes que les bailleurs de fonds mettent à la disposition du Niger. Elle se développe sous leur regard vigilant dans une arène où ils jouent, malgré tout, un rôle déterminant en raison de leur position stratégique dans le dispositif.

Cependant, l'ancrage durable de la réforme dépendra de la capacité des acteurs en présence à jouer leur rôle et à s'approprier de façon durable leurs nouvelles fonctions. Pour maintenir sa présence, l'Etat a besoin de renforcer ses compétences dans les nouvelles formes de gestion qu'il a mises en place et qui impli-

44 Le processus de décentralisation au Niger connaît un début d'exécution depuis l'installation des nouvelles communes issues des élections du 24 juillet 2005. On assiste ainsi à une nouvelle politique locale, qu'il serait nécessaire d'observer à l'avenir pour comprendre le processus d'appropriation par les communes de la gestion de l'eau. 
quent pour ses fonctionnaires une maîtrise du dispositif juridique en vigueur et des capacités accrues pour suivre la mise en œuvre de la réforme. Comme l'observe Ménard, à ce niveau, la crédibilité de l'engagement de l'autorité concédante (l'Etat) est importante puisque celle-ci doit maintenir la volonté de mener la réforme à terme, en veillant notamment à minorer la capacité d'intervention de pouvoirs discrétionnaires, en particulier ceux de l'administration ${ }^{45}$.

Quant à la Société de Patrimoine des Eaux du Niger, elle devra faire la preuve de sa capacité à gérer les biens qui lui sont concédés, en assurant son équilibre financier et en faisant en sorte qu'elle se passe des financements de l'Etat et des bailleurs de fonds et qu'elle puisse fonctionner sur la base des seules redevances qu'elle percevra de la société d'exploitation. A ce niveau, la question du prix de l'eau est déterminante puisque celui-ci conditionne les ressources financières mobilisables par la SEEN. La SPEN est par conséquent confrontée à des défis de mobilisation de ressources, de gestion et de nouveaux investissements de nature à étendre son réseau d'infrastructures hydrauliques. De sa capacité à les prendre en charge dépendra la pérennisation du nouveau dispositif. C'est d'ailleurs un des objectifs qui lui ont été clairement assignés dans la réforme.

La Société d'Exploitation des Eaux du Niger suscite pour sa part, à tort ou à raison, beaucoup de méfiance. C'est la filiale d'une multinationale réputée. Elle devra dépasser les récriminations dont elle est l'objet pour faire la preuve qu'elle est capable de promouvoir une exploitation rentable, autre que celle qui a conduit à l'effondrement de la SNE. Pour ce faire, elle doit relever le défi de créer les conditions nécessaires à l'optimisation de sa gestion (bon fonctionnement de la société, paiement par l'Etat de ses factures, paiement par les autres usagers de leurs factures, etc.) et honorer ses engagements financiers vis-à-vis de la Société de Patrimoine des Eaux du Niger.

En zone urbaine, l'Autorité de régulation multisectorielle occupe une place stratégique puisqu'il lui reviendra de garantir le respect par tous les acteurs de leur cahier des charges tel que prévu dans le contrat-plan et dans le contrat de performance annexés au contrat de concession entre la République du Niger et la SPEN. Ménard a identifié cinq caractéristiques dont la combinaison est essentielle à la capacité à limiter les possibilités de capture de l'autorité de régulation, tant de l'opérateur (SEEN) que de l'autorité concédante (Etat). Ces caractéristiques portent sur la séparation des fonctions d'efficacité et d'équité, l'autonomie politique et financière de l'organisme régulateur, la place de l'expertise dans la sélection des membres, l'identification claire de leur responsabilité et la transparence dans le processus de décision ${ }^{46}$. Il est trop tôt pour fournir des appréciations précises sur les activités de l'ARM en raison de son installation tardive. Cependant, on peut considérer qu'elle remplit déjà certaines des conditions qui la mettent à l'abri des possibilités de capture. Sans rentrer dans les détails, on relèvera plusieurs éléments qui attestent la volonté des pouvoirs publics d'assurer à cette structure quelques garanties d'indépendance: la mise en avant de la compétence technique dans le choix des membres du Conseil national de régulation, à travers notamment le recrutement des directeurs sectoriels par l'entremise d'un cabinet international; les régimes d'incompatibilité

45 C. Ménard, op. cit., p. 271.

46 C. Ménard, op. cit. 
institués par la nouvelle législation; la plénitude des attributions et les responsabilités des directeurs choisis; l'irrévocabilité des membres du Conseil national de régulation; la prestation de serment; la répartition des tâches selon les secteurs qu'elle couvre; la mise en place d'un site Internet bien fourni en documentation interne. En outre, les premières expériences de l'ARM témoignent d'une certaine vitalité si l'on en juge par la décision qu'elle a rendue relativement aux griefs portés à l'endroit de la SEEN suite à l'expertise de la gestion financière et technique de ladite société sur les exercices 2001 et $2002^{47}$.

En zone rurale, les problèmes liés à la gestion des infrastructures hydrauliques restent entiers. Il est clair que la marchandisation de l'eau concourt à l'émergence de nouveaux enjeux et de nouveaux acteurs. Ceux-ci interpellent les nouvelles communes dans leur capacité à assurer l'approvisionnement en eau potable de leurs populations et les associations d'usagers dans leur capacité à suivre les délégataires qu'elles ont choisis et à gérer les ressources issues des redevances qu'elles vont percevoir. Aux délégataires aussi se posent les problèmes liés à leur professionnalisation puisque, pour la plupart, ils s'engagent dans de nouveaux métiers. Ce ne sont là que des hypothèques qui pèsent sur le PPP tel qu'il tente de s'enraciner au Niger.

Le PPP dans le secteur de l'eau au Niger s'est mis progressivement en place depuis 2001. Sa phase actuelle va couvrir une période de dix ans, au terme desquels il sera évalué. Il marque incontestablement une nouvelle orientation dans la gestion publique de l'eau au Niger. Mais comme on l'a vu, de nombreuses hypothèques pèsent sur l'avenir du PPP. Il reviendra aux acteurs en présence de les lever s'ils veulent faire de cette réforme un outil efficace au service d'un accès à l'eau pour tous.

47 Voir Décision n ${ }^{\circ}$ D-2004/03-Ea002, sur le site Internet de l'ARM <www.arm-niger.org> 


\section{Bibliographie}

\section{Ouvrages, articles et documents consultés}

Elhadj Dagobi, A., Olivier de SARdan, J.-P., «La gestion communautaire sert-elle l'intérêt public? Le cas de l'hydraulique villageoise au Niger», Politique africaine, nº 80, 2000, pp. 153-168.

Freund B., Lootvoet, B., «Où le partenariat public-privé devient l'instrument privilégié du développement économique et local. L'exemple de Durban, Afrique du Sud», Revue Tiers Monde, $\mathrm{n}^{\circ}$ 181, janvier- mars 2005.

Hassane, A., Karadji, A., Balmer, F. (dir.), Bien gérer l'eau du Sahel. Regards sur vingt ans de coopération entre la Suisse et le Niger dans le secteur de l'hydraulique, Berne, Direction du développement et de la coopération (DDC); Niamey, Ministère de l'hydraulique et de l'environnement; Genève, IUED, 1996.

Jobert, B., Muller, P., L'Etat en action. Politiques publiques et corporations, Paris, Presses Universitaires de France, 1987.

LAImé, M., «Main basse sur l'eau des villes », Le Monde diplomatique, mars 2005, pp. 16-17.

Le Monde: Dossiers et Documents, «Saga Vivendi Universal: un rêve en échec», $\mathrm{n}^{\circ}$ 320, mai 2003.

MÉNARD, C., «Enjeux d'eau: la dimension institutionnelle», Revue Tiers Monde, n 166, avril-juin 2001, pp. 261-274.

Ministère De L'HYdRAUliQue ET DE L'EnVIRONnEMENT, Politiques et stratégies pour l'eau et l'assainissement. De l'eau pour un développement durable, Niamey, mai 2001.

MINISTÈRE DES FINANCES ET DU PLAN, Assemblée générale des entreprises publiques et parapubliques, rapport général, mars 1996.

Plane, P., «La privatisation de l'électricité en Côte d'Ivoire : évaluation et interprétation des premiers résultats », Revue Tiers Monde, n 152, octobre-décembre 1997.

SidiBÉ, S., La réforme du secteur parapublic au Niger, Niamey, Nouvelle Imprimerie du Niger, n.d.

SociÉTÉ D'EXPLOITATION DES EAUX DU NigER, Assistance du maître d'ouvrage «SPEN» à la réalisation des bornes fontaines, rapport final, septembre 2004.

\section{Textes législatifs et réglementaires}

Contrat de concession entre la République du Niger et la Société de Patrimoine des Eaux du Niger (SPEN), mars 2001.

Décision n ${ }^{\circ}$ D- 2004/03-Ea002, disponible sur le site Internet de l'ARM, <www.arm-niger.org>.

Loi 2000-12 du 14 août 2000, portant réorganisation de l'activité de production, transport et distribution de l'eau dans le sous-secteur de l'hydraulique urbaine et créant la Société de Patrimoine des Eaux du Niger (SPEN).

Ministère de L'hydraulique et de l'environnement, Programme hydraulique Suisse Niger, Le rôle de l'Etat dans la gestion des ressources naturelles. Recueil des textes législatifs et réglementaires, Niamey, 1999.

MINISTÈRE DU PLAN, MINISTÈRE DES RESSOURCES EN EAU, Lettre de politique sectorielle de l'hydraulique urbaine, Niamey, 08/03/2001.

Ordonnance $n^{\circ}$ 93-014 du 2 mars 1993 portant régime de l'eau, modifiée par la Loi n ${ }^{\circ}$ 98-041 du 7 décembre 1998.

Ordonnance $\mathrm{n}^{\circ}$ 96-062 du 22 octobre 1996.

Ordonnance n ${ }^{\circ}$ 99-044 du 26 octobre 1999 portant création, organisation et fonctionnement d'une autorité de régulation multisectorielle.

\section{Site Internet}

Autorité de régulation multisectorielle (ARM) : <www.arm-niger.org $>$.

\section{Presse écrite consultée}

Alternative Magazine

Le Démocrate

Le Régulateur

Le Républicain

Sahel Dimanche 